\title{
NOTE RECTIFICATIVE SUR LA TERMINOLOGIE UTILISÉE DANS NOTRE CLASSIFICATION
}

\section{DES RHINONYSSIDAE (ACARINA-MESOSTIGMATA)}

\author{
Par A. FAIN
}

Nous avons proposé précédemment $(1956,1957 a, 1957 b)$ une nouvelle classification des Acariens de la famille Rhinonyssidx basée essentiellement sur la structure des chélicères.

Dans ces études, nous avons utilisé le terme de "péritrème » dans un sens plus large que celui qu'on lui attribue généralement.

Nous avons dit récemment en parlant du stigmate chez les Rhinonyssidæ: "Le stigmate est entouré d'un simple anneau chitineux ou d'une membrane transparente, ponctuée, arrondie ou allongée (péritrème). $\$(1957 b$, p. 39). Dans nos travaux précédents, nous avons donc donné le nom de «péritrème » à la membrane allongée entourant le stigmate et le petit canal qui le prolonge et, lorsque celui-ci fait défaut, à la membrane circulaire ou ovalaire entourant seulement l'orifice stigmatique.

Rappelons que, chez les Rhinonyssidx, le stigmate est prolongé en avant par un étroit et court canal chitineux qui est classiquement appelé «péritrème ». Ce canal est souvent très court et, dans certains cas même, il peut faire complètement défaut. Chez certaines espèces, la longueur de ce petit canal est difficile à apprécier avec exactitude et c'est la raison pour laquelle nous avons remplacé ce caractère par un autre, qui est celui de la forme de la membrane chitinisée qui entoure l'orifice stigmatique, ainsi que le petit canal qui en part.

Pour dissiper toute équivoque, nous rappellerons ici sous quels aspects peut se présenter l'orifice stigmatique dans les différents genres de la famille Rhinonyssidæ:

$1^{\circ}$ Le stigmate n'est pas prolongé par un petit canal (absence de «péritrème » au sens classique). Dans ce cas l'orifice stigmatique est entouré seulement d'un étroit anneau chitineux (genres Rhinonyssus et Sternostoma), ou bien d'une membrane

Ann. de Parasitologie, t. XXXIV, $\mathrm{n}^{\circ} 1-2 .-1959$. 
chitinisée circulaire ou ovalaire (genres Rhinonyssus, Sternostoma et Ptilonyssus).

$2^{\circ}$ Le stigmate est prolongé par un petit canal (= péritrème au sens classique). Dans ce cas il existe une membrane chitinisée allongée qui entoure le stigmate et le petit canal en question (Genres Ptilonyssus, Neonyssus, Rallinyssus, Larinyssus, Astridiella, Rhinoecius, Ruandanyssus).

La définition élargie du mot «péritrème » utilisée par nous dans les travaux cités ci-dessus pouvant donner lieu à des interprétations erronées, nous avons décidé de l'abandonner et de nous en tenir désormais à la définition classique de ce terme.

Voici donc comment se présentera la nouvelle classification des Rhinonyssidæ que nous avons proposée en 1956 et 1957 , après y avoir introduit cette modification relative à la définition du péritrème.

\section{Définition de la famille RHINONYSSIDA}

(Trouessart, 1895), Vitzthum, 1935

Acariens de taille moyenne ou relativement grande (entre 385 et $1.600 \mu$ de long), munis d'écussons chitineux peu sclérifiés, en nombre variable, typiquement 6 (3 ventraux : sternal, génital et anal ; et 3 dorsaux : podosomal, opisthosomal et pygidial). Pattes bien développées, toutes terminées par un prétarse portant une paire de griffes et une ventouse. Stigmate situé dans la région du podosoma ou en arrière du corps ; dans le premier cas, il est dorsal ou dorsolatéral. Le stigmate présente un court péritrème dirigé vers l'avant ou est dépourvu de péritrème. Chélicères relativement courts, avec doigts très courts ou relativement longs chez la femelle; chez le mâle, les doigts sont toujours relativement longs. Palpes à 4 articles. Tritosternum présent ou non. Chætotaxie généralement réduite. Parasites des fosses nasales des Oiseaux.

Genre type : Rhinonyssus Trouessart, 1894.

\section{Classification des RHINONYSSIDA (femelles)}

1) Sous-Famlle Rhinonyssinæ (Trouessart, 1895), Fain, emend.

Diagnose: Chélicères terminés par deux doigts relativement longs, dont la longueur atteint au moins le cinquième ou le sixième de la longueur totale des chélicères. Ces doigts sont toujours beaucoup plus longs que larges. Le stigmate est situé, soit dans la région du podosoma, et dans ce cas il est dépourvu de péritrème ou au contraire possède un 
court péritrème dirigé vers l'avant, soit dans la région postérieure du corps et il se prolonge alors par un court péritrème. Griffe I généralement peu modifiée, parfois normale ou de taille plus petite ou plus grande que les suivantes. Gnathosoma terminal ou en partie ventral. Absence de tritosternum. Un, deux ou trois écussons dorsaux principaux.

\section{Genres :}

1. Rhinonyssus Trouessart, 1894.

Péritrème absent. L’orifice stigmatique, situé dans la région podosomale, est entouré soit d'un simple anneau chitineux, soit d'une petite membrane chitinisée arrondie d'aspect ponctué.

Doigt mobile du chélicère atteignant au moins le $5^{-}$de la longueur totale du chélicère, sans très forte dent préapicale.

EsPÈcE TYPE : Rhinonyssus coniventris Tr., 1894.

\section{Neonyssus Hirst, 1921.}

Le stigmate est situé dans la région podosomale et se prolonge par un court péritrème. Stigmate et péritrème sont entourés d'une membrane chitineuse elliptique d'aspect ponctué.

Doigt mobile du chélicère comme dans le genre Rhinonyssus.

EsPÈCE TYPE : Neonyssus intermedius Hirst, 1921.

3. Rallinyssus Strandtmann, 1948.

Le stigmate, muni d'un péritrème, a la même structure que dans le genre Neonyssus, mais il est situé à la partie postérieure du corps.

Doigt mobile du chélicère comme dans le genre Rhinonyssus, mais relativement plus long que dans ce genre.

EsPÈCE TYPE : Rallinyssus caudistigmus Strandtmann, 1948.

4. Larinyssus Strandtmann, 1948 .

Stigmate comme dans le genre Neonyssus.

Doigt mobile portant une très forte dent préapicale.

EsPÈCE TYPE : Larinyssus orbicularis Strandtmann, 1948.

\section{II) Sous-famille Ptilonyssinæ (Castro, 1948), Fain, emend.}

Diagnose : Chélicères terminés par deux doigts très courts n'atteignant pas le dixième de la longueur totale du chélicère, souvent beaucoup plus courts. Doigt mobile triangulaire à base postérietire, presque aussi large que long. Stigmate situé dans la région du podosoma, avec ou sans péritrème. Gnathosoma terminal ou complètement ventral, avec tous les intermédiaires. Tritosternum présent ou absent. Griffes I généralement modifiées. Présence d'un, de deux ou de trois écussons dorsaux principaux. 


\section{Genres :}

1. Sternostoma Berlese et Trouessart, 1889.

Stigmate sans péritrème, entouré d'un simple anneau chitineux ou d'une membrane ponctuée arrondie ou légèrement ovalaire. Gnathosoma complètement ou partiellement ventral. Il n'y a jamais d'écusson pygidial chez l'adulte. Les quelques nymphes examinées ne montrent pas d'écusson pygidial, ni de fortes épines pygidiales. Absence de tritosternum. Coxæ I souvent très développées et pouvant se toucher sur la ligne médiane.

EsPÈce TYPE: Sternostoma cryptorhynchum Berl. et Tr., 1889.

2. Ptilonyssus Berlese et Trouessart, 1889.

Stigmate généralement avec péritrème. Lorsqu'il n'y a pas de péritrème, l'orifice stigmatique est entouré simplement d'une membrane ponctuée arrondie ou légèrement ovalaire. Les trois espèces dépourvues de péritrème possèdent un écusson pygidial. Chez la nymphe, le péritrème est probablement constant. Ecusson pygidial manquant rarement chez la femelle et probablement constant chez la nymphe, cette dernière portant deux fortes épines pygidiales légèrement barbelées. Gnathosoma terminal. Pas de tritosternum.

Espèce Type : Ptilonyssus echinatus Berl. et Tr., 1889.

3. Astridiella Fain, 1957.

Stigmate avec péritrème. Gnathosoma terminal. Pas d'écusson pygidial. Tritosternum présent.

EsPĖce TYPE : Astridiella scotornis Fain, 1956.

\section{III) Sous-Famille Rhinoeciinæ Fain, 1957.}

Diagnose : Chélicères terminés par un seul doigt, le doigt fixe faisant défaut. Tritosternum présent ou absent. Stigmate situé dans la région du métapodosoma, possédant un péritrème. Griffes I peu ou pas modifiées. Gnathosoma terminal ou subterminal.

\section{Genres :}

1. Rhinoecius Cooreman, 1946.

Pas de tritosternum. Il y a trois paires de poils sternaux. Stigmate dorsal.

ESPÈCE TYPE : Rhinoecius oti Coor., 1946.

2. Ruandanyssus Fain, 1957.

Tritosternum présent. Il y a trois ou quatre paires de poils sternaux. Stigmate dorsal ou dorso-latéral.

EsPÈCE TYPE : Ruandanyssus terpsiphonei Fain, 1957. 


\section{Clé de la famille des RHINONYSSIDAE (femelles)}

1. Chélicères terminés par un seul doigt, le doigt fixe faisant

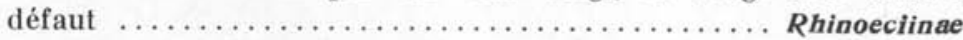
Chélicères terminés par deux doigts, un fixe et un mobile .....

2. La longueur des doigts atteint au moins le cinquième ou le sixième de la longueur totale du chélicère ...... Rhinonyssinae La longueur des doigts n'atteint pas le dixième de la longueur totale du chélicère $\ldots \ldots \ldots \ldots \ldots \ldots \ldots \ldots$ Ptilonyssinae

3. Tritosternum absent, trois paires de poils sternaux . Rhinoecius Tritosternum présent, trois à quatre paires de poils sternaux ..

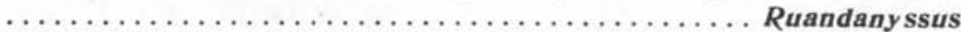

4. Stigmate situé dans la partie postérieure du corps ...Rallinyssus Stigmate situé dans la région du podosoma $\ldots \ldots \ldots \ldots \ldots \ldots \ldots$

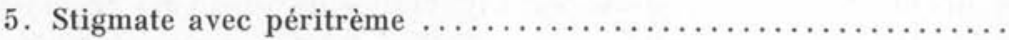
Stigmate sans péritrème $\ldots \ldots \ldots \ldots \ldots \ldots \ldots$ Rhinonyssus

6. Doigt mobile avec une très forte dent préapicale ..... Larinyssus Pas de très forte dent préapicale au doigt mobile ..... Neonyssus

7. Stigmate sans péritrème. Pas d'écusson pygidial ni de tritosternum $\ldots \ldots \ldots \ldots \ldots \ldots \ldots \ldots \ldots \ldots \ldots \ldots \ldots \ldots \ldots \ldots \ldots \ldots$ Sternostoma Stigmate avec péritrème ou sans péritrème mais dans ce cas il

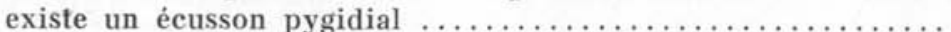

8. Stigmate avec péritrème. Tritosternum présent. Pas d'écusson

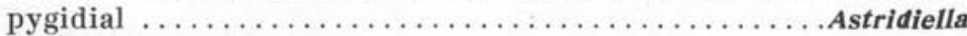

Stigmate généralement avec péritrème. Lorsqu'il n'y a pas de péritrème l'orifice stigmatique est entouré d'une membrane ponctuée circulaire, ou ovalaire. Tritosternum absent ....Ptilonyssus

\section{BIBLIOGRAPHIE}

FAIN (A.), 1956. - Les Acariens de la famille Rhinonyssidxe Vitzthum 1935, parasites des fosses nasales des Oiseaux au Ruanda-Urundi (Note préliminaire). Rev. Zool. Bot. Afr., LIII (1-2), 131-157.

FAIN (A.), 1957a. - Essai de classification des Rhinonysside (Acari : Mesostigmata) avec description de deux genres nouveaux. Ann. Parasitologie, XXXI (1-2), 145-157.

FAIN (A.), 1957b. - Les Acariens des familles Epidermoptide et Rhinonysside, parasites des fosses nasales d'Oiseaux au Ruanda-Urundi et au Congo Belge. Ann. Mus. Royal du Congo Belge, Tervuren, Série 8, 60, 1-176.

\section{Institut de Médecine Tropicale d'Anvers}

Laboratoire de Zoologie Médicale 